\title{
Probing defects in nanostructures with high spatial and energy resolution
}

\section{Nasim Alem}

\section{Pennsylvania State University, Washington, District of Columbia, United States}

Defect, dopants, and interfaces have a profound effect on the physical, chemical, and electronic properties of nanostructures by modifying their local electronic structure, modulating their band gap, and perturbing the charge distribution within their vicinity. Complex defects can form as a result of vacancy-dopant coupling which can further perturb the atomic and electronic structure and the spatial charge distribution within their vicinity. Such perturbations have shown to have a profound impact on the local electronic structure of the crystal and have given rise to novel and transformative photonic, electronic, and optoelectronic properties of a wide range of nanostructures including $2 \mathrm{D}$ crystals and wide band gap oxides, among other systems.

Using high resolution aberration-corrected scanning/transmission electron microscopy imaging and electron energy loss spectroscopy, we investigate the atomic and chemical structure of the defects and their impact on the physical and electronic properties of nanostructures. In the family of 2D crystals, i.e. graphene and Transition Metal Dichalcogenides (TMDs), intrinsic defects can arise during the synthesis process [1,2]. Such defects can act as the nucleation sites during heterostructure growth and further influence the atomic and electronic structure of the resulting heterostructures. Figure 1 shows an ADFSTEM image of a twin boundary in a monolayer $\mathrm{WSe}_{2}$ crystal as an example of a defect array resulting from synthesis. Besides the defects, interfaces in 2D crystal heterostructures can maintain peculiar atomic structures and novel properties when dissimilar 2D crystal building blocks, such as MoS2 and ReS2 are put together. This talk will present how defects and interfaces in TMD heterostructure family, i.e. $\mathrm{MoS}_{2}$, $\mathrm{WS}_{2}, \mathrm{WSe}_{2}, \mathrm{ReS}_{2}$, and graphene, can lead to atomic reconstruction and further modify the local electronic structure in the $2 \mathrm{D}$ crystal heterostructures.

In the family of wide band gap materials, beta- $\mathrm{Ga}_{2} \mathrm{O}_{3}$ is of particular interest in high power electronics and photodetection due to having a high breakdown field and wide band gap [3]. Here, we explore how dopants and interstitial atoms in beta- $\mathrm{Ga}_{2} \mathrm{O}_{3}$ can lead to the formation of complex defects and further modify its local electronic structure (Fig. 2). beta- $\mathrm{Ga}_{2} \mathrm{O}_{3} /$ beta- $\left(\mathrm{Al}_{\mathrm{x}} \mathrm{Ga}_{1-\mathrm{x}}\right)_{2} \mathrm{O}_{3}$ interfaces are particularly interesting due to showing a high electron mobility and the presence of $2 \mathrm{D}$ electron gas. Using ADFSTEM imaging combined with EELS we further uncover the interfacial structure of this crystal, the role of interstitial atoms and dopants in modulating the band gap, and the presence of 2DEG and a higher electron mobility at the interface in beta- $\mathrm{Ga}_{2} \mathrm{O}_{3} /$ beta- $\left(\mathrm{Al}_{\mathrm{x}} \mathrm{Ga}_{1-\mathrm{x}}\right)_{2} \mathrm{O}_{3}$ interfaces. 

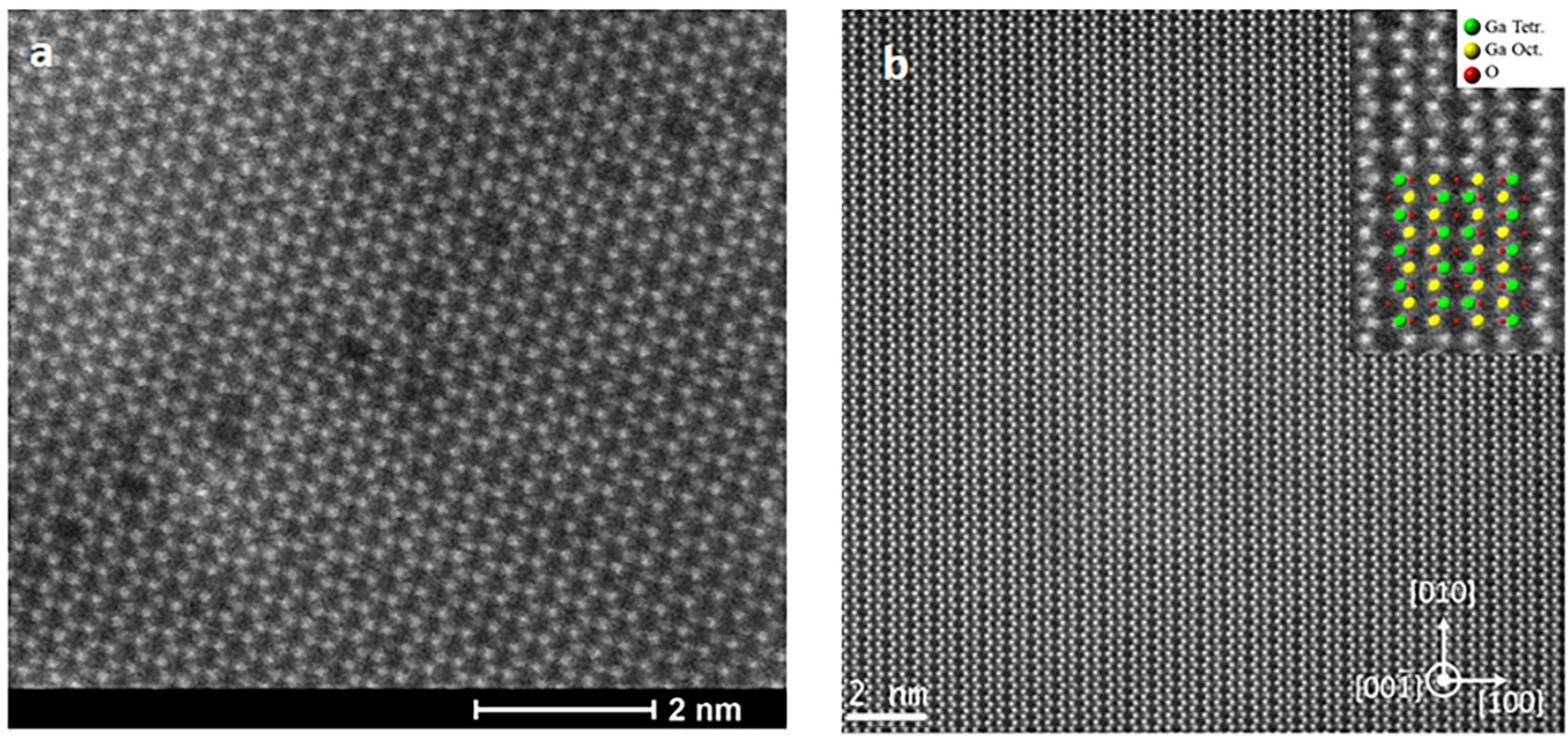

Figure 1. Figure 1. (a) ADF-STEM image of (a) a twin boundary in a monolayer WSe2 crystal synthesized by MOCVD process, and (b) a defect-free $\beta-\mathrm{Ga} 2 \mathrm{O} 3$ crystal imaged along the [001] zone with octahedral and tetrahedral sites marked on the inset.

\section{References}

[1] D. R. Hickey et al., arXiv e-prints 2020, arXiv: 2006.1166 (2020)

[2] D. R. Hickey et al., 2D Materials 2020, 8, (1), 011003 (2020)

[3] S. J. Pearton et al., Appl Phys Rev, 5, (1), 011301 (2018)

[4] This work was supported by the National Science Foundation (NSF), in part under the CAREER program (DMR-1654107), in part by the program EFRI 2-DARE: 2D Crystals by Activated Atomic Layer Deposition (EFRI-1433378), and in part by the Penn State 2D Crystal Consortium-Materials Innovation Platform (2DCC-MIP) under NSF cooperative agreement DMR-1539916. The work on the wide bang gap systems at PSU was supported by the Air Force Office of Scientific Research (AFOSR) program FA9550-18-1-0277 as well as GAME MURI, 10059059-PENN. This work utilized resources provided by the NSF-MRSEC-sponsored Materials Characterization Lab at Penn State. 